\title{
TUMOR HEPÁTICO EXPERIMENTAL (VX-2) EM COELHO. IMPLANTAÇÃO DO MODELO NO BRASIL ${ }^{1}$
}

Rogério Saad Hossne ${ }^{2}$

\begin{abstract}
Hossne RS. Tumor hepático experimental (VX-2) em coelho: implantação do modelo no Brasil. Acta Cir Bras [serial online] 2002 Jul-Ago;17(4). Disponível em URL: http://www.scielo.br/acb.

RESUMO - Os estudos para a investigação de novas modalidades terapêuticas em biologia tumoral, deveriam passar por estudos experimentais prévios. Neste sentido dispõem-se hoje de uma grande variedade de modelos tumorais experimentais; em determinadas investigações faz-se necessária a adequação do modelo tumoral às necessidades biológicas, patológicas e experimentais dos estudos. Desta forma, em nosso serviço, buscávamos um modelo tumoral hepático para estudos experimentais que se adequasse às seguintes características: fácil manipulação, crescimento controlável, evolução e agressividade semelhantes aos seres humanos. Os dados da literatura nos levaram a busca do tumor hepático VX-2, em coelhos. Neste artigo discutimos as vantagens da utilização deste modelo experimental e a sua introdução em nosso país.
\end{abstract}

DESCRITORES - Fígado. Neoplasias hepáticas. Modelos biológicos. Coelhos.

\section{INTRODUÇÃO}

A qualidade dos estudos na fase pré-clínica, passo essencial para a correta aplicação dos ensaios clínicos em seres humanos, depende muito da adequação dos modelos em animais.

$\mathrm{Na}$ área da oncologia, diversos modelos experimentais têm sido propostos, buscando atender a esse objetivo.

De modo geral, quer para os estudos básicos de biologia tumoral, quer para a investigação de novas modalidades terapêuticas (cirúrgica, medicamentosa, radioterápica) dispõem-se hoje de uma variedade de tumores experimentais, já catalogados.

Em nosso meio, três modelos têm sido mais utilizados: tumor ascítico de Ehrlich, tumor de Walker e o melanoma B16 (menos freqüentemente).

O tumor de Walker, largamente utilizado em patologia tumoral, tem como hospedeiro o rato e as suas principais características são: crescimento e evolução rápidas e de difícil controle, lesões múltiplas em tecidos sólidos e alta agressividade. Essas características dificultam a padronização de técnica adaptada a projetos experimentais quando se necessita de modelos menos agressivos, com lesões únicas e de crescimento controlado.

O tumor de Ehrlich, em camundongos, quando comparado ao de Walker, é menos agressivo quanto ao crescimento tumoral, sendo este definido pela dose de células transplantadas, o que permite, assim, certo grau de controle; desenvolve-se em cavidades serosas como a abdominal e pleural, bem como em músculos; não se observa, porém, crescimento em vísceras parenquimatosas, como fígado. O tumor de Ehrlich é bom modelo para estudos envolvendo citologia, por crescer na forma ascítica, o que permite a obtenção de células tumorais isoladas.

O melanoma B16 é bom modelo para estudos de tumores metastáticos, tendo afinidade quase que exclusiva para o tecido pulmonar. O tumor pode ser mantido na forma de cultura de células, sendo implantado no tecido celular subcutâneo em ratos (Black, Edwards), com grande capacidade para metastatizar para os pulmões. Apresenta 2 sub-clones principais: G.35 de crescimento lento e G.3.12. de crescimento rápido, ambos provenientes da sublinha $\mathrm{F} 10^{1}$.

1. Trabalho realizado no Laboratório de Técnica Cirúrgica e Cirurgia Experimental "William Saad Hossne", Faculdade de Medicina de Botucatu - UNESP (FM-UNESP).

2. Professor Doutor do Departamento de Cirurgia e Ortopedia da FM - UNESP. 
Os três modelos têm sido amplamente utilizados com êxito, desde que haja adequação entre as características biológicas tumorais e a proposta de investigação.

Em determinadas investigações experimentais de tumores hepáticos (isolados ou múltiplos), uma das dificuldades é a produção de uma lesão que tenha crescimento relativamente lento e controlável, que apresente características patológicas (macro e micro), bem como evolução e agressividade que se assemelhem ao que ocorre em situações clínicas. Em experimentação de natureza cirúrgica, pode ser também fator importante o porte do animal, de modo a facilitar ou mesmo possibilitar a intervenção cirúrgica em estudo, bem como existir tempo de sobrevida suficiente para permitir a avaliação das variáveis em estudo.

Neste sentido, procuramos padronizar em nosso meio o modelo experimental de tumor hepático com algumas dessas características, que não são atendidas pelos modelos do Ehrlich, do Walker e do B16.

Os dados da literatura nos levaram à busca do tumor hepático VX-2, em coelhos.

Em 1938, nos laboratórios do Instituto Rockefeller para Pesquisas Médicas, conjuntamente com o Departamento de Cirurgia da Universidade Médica de Cornell, após transformação maligna de papiloma induzida por vírus, obtiveram um carcinoma de células escamosas ${ }^{2}$.

O carcinoma foi progressivamente sendo implantado na musculatura de coelhos, com sucesso, conseguindo-se a padronização e a manutenção deste modelo tumoral. Do ponto de vista anatomopatológico, tratase de adenocarcinoma difuso e pouco diferenciado (Figura 1).

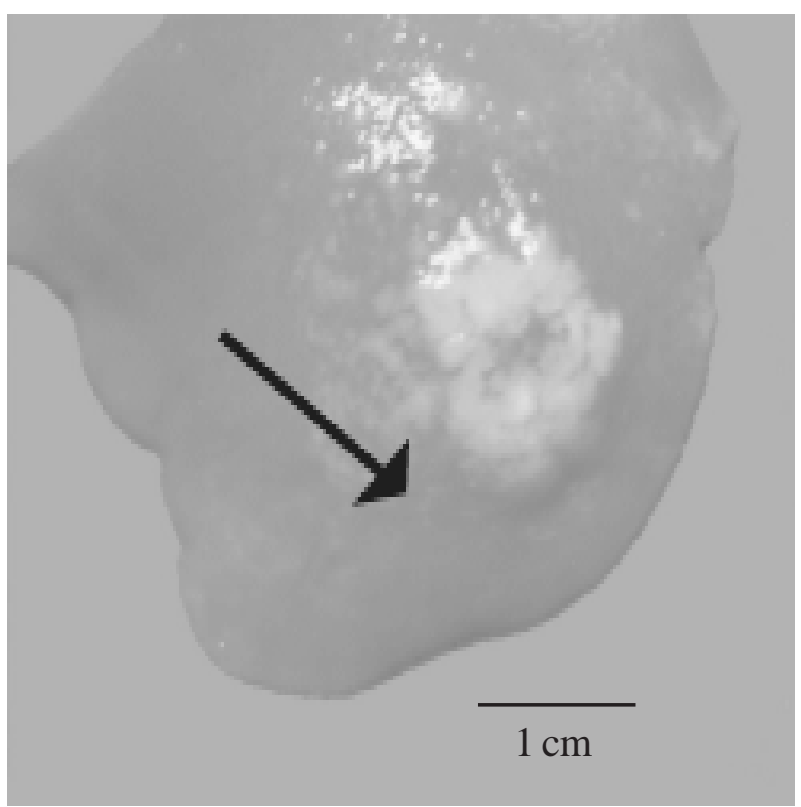

Figura 1 - Aspecto macroscópico do fígado de coelho portador do tumor VX-2, decorridos 7 dias da infiltração de suspensão de células tumorais.
O tumor VX-2 pode ser implantado em qualquer estrutura orgânica com boa vascularização e, de preferência, sólida; os órgãos mais utilizados são músculos, fígado, baço e ossos ${ }^{3}$; apresenta como característica o crescimento uniforme, circunferencial, dose dependente (Figura 2).

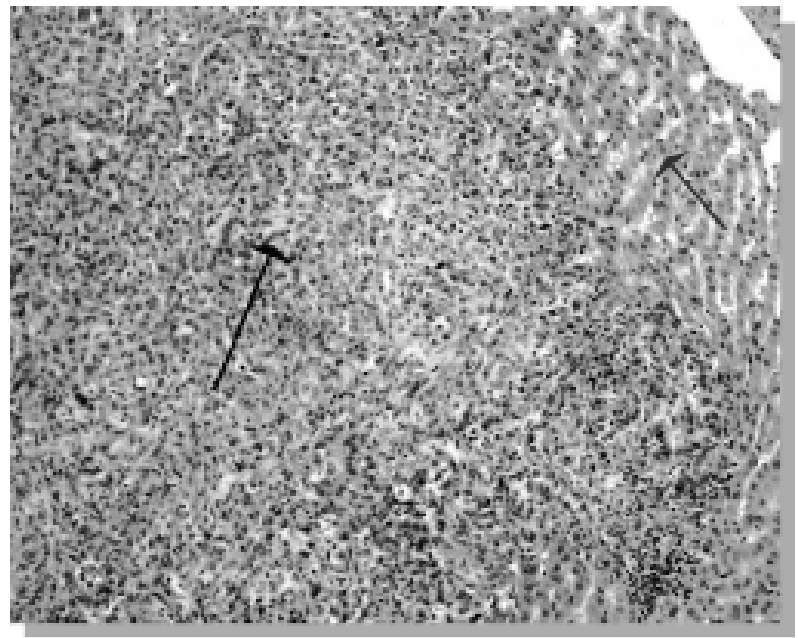

Figura 2 - Corte histológico do fígado de coelho portador do tumor VX-2, decorridos 7 dias da infiltração de suspensão de células tumorais. Notar o extenso nódulo tumoral (seta preta) com parênquima hepático normal adjacente (seta azul). X200. Coloração HE.

Do ponto de vista técnico, obtém-se a suspensão de células após a maceração do tecido implantado (fígado/músculo) com meios de cultura; a seguir, é feita a análise da viabilidade e a contagem das células da suspensão, determinando-se o número de células a ser injetado de acordo com as necessidades do pesquisador.

A análise da literatura evidencia ampla difusão e utilização deste tumor, possivelmente em decorrência de suas características biológicas, favoráveis a estudos experimentais de média duração.

O tumor VX-2 tem sido empregado em estudos radiológicos de detecção de tumor, objetivando técnicas que aumentem a sensibilidade dos meios diagnósticos, como tomografia ${ }^{4,5}$ e ressonância nuclear magnética ${ }^{6,7}$, estudos com PET scan e no desenvolvimento de drogas quimioterápicas ${ }^{9,10,11}$ bem como em outras modalidades de tratamento como quimioembolização ${ }^{7,12}$ e radiofreqüência.

Após várias tentativas e contatos com diversos laboratórios dos EUA e Japão, conseguimos obter em junho de 1999 junto a Brian Olliver, MD da Universidade de Boston, três amostras congeladas do tumor VX-2, com o objetivo de implantar o modelo no Laboratório de Cirurgia Experimental do Departamento de Cirurgia e Ortopedia da Faculdade de Medicina de Botucatu - UNESP. 


\section{MÉTODOS}

Após o descongelamento e contagem das células tumorais, procedemos ao implante do tumor na musculatura da pata inferior esquerda e no fígado de 2 coelhos machos. Decorridos 5 dias, notamos o crescimento de uma lesão sólida na musculatura dos animais; ao final de 10 dias, a lesão apresentava $3 \mathrm{~cm}$ de diâmetro. Nesta ocasião, observamos as mesmas características da lesão no fígado. Após a maceração destes tecidos em separado, obtivemos nova suspensão de células tumorais que, após a contagem e verificação da viabilidade, foram injetadas em novos animais (coelhos) sadios.

Atualmente, mantemos este tumor com repiques a cada 10 dias.

\section{DISCUSSÃO}

Com esta sistemática técnica, tem sido possível produzir lesões hepáticas que simulam lesão isolada ou metastáticas múltiplas observadas em situação clínica.

No momento, estamos iniciando estudos sobre ação de drogas, em tumor hepático VX2. Em breve, teremos condições de fornecer cepa tumoral aos pesquisadores nacionais interessados no modelo experimental.

\section{REFERÊNCIAS}

1. Alterman A, Fornabaio DM, Stackpole C. Metastatic dissemination of B16 melanoma: pattern and sequence of metastasis. J N CI 1985;4:691-9.
2. Peyton R, Kidd JG, Smith WE. Experiments on the cause of the rabbit carcinomas derived from virus-induced papillomas. $4^{\text {th }}$ International Cancer Congress, St. Louis, September, 1947.

3. Enneking WF, Fkynn L. Effects of VX-2 carcinoma implanted in bone in rabbits. Cancer Res 1968;28:1007-3.

4. De Baere T, Zhang X, Aubert B. Quantification of tumor uptake of iodized oils and emulsions of iodized oils: experimental study. Radiology 1996;201:731-6.

5. Lenader P. An imaging study in a rabbit tumor model. Acta Radiol 1996;37:63-8, 1996.

6. Yamada K, Jimbo T, Miyahara K. Contrast effect of DV6807 in angiography and CT in experimental animal model. J Vet Med Sci 1996;58:389-96.

7. Pauser S, Wagner S, Lippmann P. Evaluation of efficient chemoembolization by magnetic resonance: an experimental study on the VX-2 tumor in the rabbit liver. Cancer Res 1996;56:1863-7.

8. Oya N, Nagata Y, Tamaki N. Evaluation of experimental liver tumors using fluorine $-18-2$ - fluoro PET. J Nuclear Med 1996;37:296-302.

9. Izumi B,Tashiro S, Miyauchi Y. Anticancer effects of local administration of mitomycin-C via artery on portal vein on implantation and growth of VX-2 cancer injection in rabbit liver. Cancer Res 1986;46:4167-70.

10. Zharo Z, Ramiry LN, Simenauer N. Pharmacokinectis of intra-arterial hepatic THP-adnamycin-lyprodol in rabbits with VX-2 tumor. Reg Cancer Treat 1993;4:213-7.

11. Iwai K, Maeda H, Konno T. Tumor targeting by arterial administration of lipids: rabbit model with VX-2 carcinoma in the liver. Anticancer Res 1987;7:321-8.

12. Beppu T, Ohara C, Yamaguchi Y. A new chemsembolization therapy in rabbits liver with VX-2. Reg Cancer Treat 1992;5:33-5.

Hossne FS. Experimental liver tumor (VX-2) in rabbits: implantation of the model in Brazil. Acta Cir Bras [serial online] 2002 Jul-Aug;17(4). Available from URL: http://www.scielo.br/acb.

ABSTRACT - Studies for investigation of new therapeutic modalities in tumoral biology should be based on previous experimental studies. Then, there are a great variety of tumoral experimental models today. Some investigations have been done necessary an adaptation of the tumoral model to the needing of the studies biological and pathological. So, in our laboratory, we looked for a tumoral hepatic model for experimental studies with the following characteristics: easy manipulation, control of growing, evolution and aggressiveness like to humans. Data of the literature took us the search of the hepatic tumor VX-2, in rabbits. In this article we discussed the advantages of use this experimental model and its introduction in our country. Experimental hepatic tumor (VX-2) in rabbit. Implantation of the model in Brazil.

KEY WORDS - Liver. Liver neoplasms. Models, biological. Rabbits.

\section{Endereço para correspondência:}

Rogério Saad Hossne

Departamento de Cirurgia e Ortopedia

Faculdade de Medicina de Botucatu - UNESP

Distrito de Rubião Júnior, s/n

18618-970 Botucatu - SP

Tel: (14) 6824-6170 / 9775-1179
Conflito de interesse: nenhum Fonte de financiamento: nenhuma
Data do recebimento: 03/02/2002

Data da revisão: 22/02/2002 Data da aprovação: 18/03/2002 